\title{
Is an obscured AGN at the centre of the disk galaxy IC 2497 responsible for Hanny's Voorwerp?
}

\author{
H. Rampadarath ${ }^{*} a{ }^{\dagger}$, M.A. Garrett ${ }^{b c e}$, T. Muxlow ${ }^{d}$, G. I. G. Józsa ${ }^{b}$, T. A. Oosterloo ${ }^{b f}$, \\ and Z. Paragi ${ }^{a g}$ \\ a Joint Institute for VLBI in Europe (JIVE), The Netherlands \\ ${ }^{b}$ Netherlands Institute for Radio Astronomy (ASTRON), The Netherlands \\ ${ }^{c}$ Sterrewacht Leiden, Leiden University, The Netherlands \\ ${ }^{d}$ Jodrell Bank Centre for Astrophysics, The University of Manchester, UK \\ ${ }^{e}$ Centre for Supercomputing, Swinburne University of Technology, Australia \\ ${ }^{f}$ Kapteyn Astronomical Institute, University Groningen, The Netherlands \\ ${ }^{g}$ MTA Research Group for Physical Geodesy and Geodynamics, Hungary \\ E-mail: rampadarathejive.nl, garretteastron.nl
}

\begin{abstract}
We present the results of VLBI and MERLIN observations of the massive disk galaxy IC 2497. Optical observations of IC 2497 revealed the existence of a giant emission nebula "Hanny's Voorwerp" in the proximity of the galaxy. Earlier short-track $18 \mathrm{~cm}$ observations with e-VLBI at $\lambda$ $18 \mathrm{~cm}$, detected a compact radio component (C1) at the centre of IC 2497. The brightness temperature of $\mathrm{C} 1$ was measured to be $>4 \times 10^{5} \mathrm{~K}$. Deeper, long-track e-VLBI observations presented here, re-confirm the existence of $\mathrm{C} 1$ but also reveal the existence of a second compact component (C2) located about 230 milliarcseconds to the North-East of $\mathrm{C} 1$. The brightness temperature of $\mathrm{C} 2$ is measured to be $>1.4 \times 10^{5} \mathrm{~K}$, suggesting that both components may be related to AGN activity (e.g. a radio core and jet hotspot). Lower resolution $\lambda 18 \mathrm{~cm}$ MERLIN observations show both components. $\mathrm{C} 1$ is shown to be compact with a slight elongation along the direction of Hanny's Voorwerp, and C2 shows a lot of extended emission in an almost perpendicular direction to the direction of the Voorwerp. Our results continue to support the hypothesis that IC 2497 contains an Active Galactic Nucleus (AGN), and that a jet associated with this AGN clears a path that permits ionising radiation from the AGN to directly illuminate the emission nebula.
\end{abstract}

Science and Technology of Long Baseline Real-Time Interferometry:

The 8th International e-VLBI Workshop, EXPReSO9

June 22 - 262009

Madrid, Spain

\footnotetext{
*Speaker.

${ }^{\dagger}$ Currently at The School of Physics and Astronomy, University of Manchester, UK
} 


\section{Introduction}

In early 2008, Dutch school teacher, Hanny van Arkel, discovered one of the most bizarre objects to come out of the GalaxyZoo.org morphological census [1], SDSS J094103.80

+344334.2. This object, now known as "Hanny's Voorwerp" ${ }^{1}$ [1] appears as an irregular cloud located $15-25 \mathrm{kpc}$ to the South-East of the massive disk galaxy IC 2497 [1,2]. Optical observations indicate a high-ionisation state of the cloud despite the lack of a stellar counterpart [1]. The quiescent kinematics as derived from optical spectra suggest ionisation from photons as the predominant ionisation process, rather than ionisation from shocks [1, 2].

Recent radio observations using the Westerbork Synthesis Telescope (WSRT) [2], has shown the existence of a radio continuum source at the central position of IC 2497, with an extension in the direction of Hanny's Voorwerp. In addition, neutral hydrogen is detected around the galaxy and the Voorwerp is probably part of this large gas reservoir. HI is also detected in absorption towards the central radio core. Clearly, obscuring material in the direction of the core of IC 2497 is present, while the extended continuum points towards the presence of a radio jet.

Here we present results of new e-VLBI and MERLIN observations of IC 2497 to investigate the small-scale structure of the core of IC 2497.

\section{Observations and Initial Results}

\section{1 e-VLBI observations}

IC 2497 is a disk galaxy with a redshift of $\mathrm{z}=0.050221$ [4] at R.A. $=09 \mathrm{~h} 41 \mathrm{~m} 04.094 \mathrm{~s}$ and Dec. $=+34 \mathrm{~d} 43 \mathrm{~m} 58.03 \mathrm{~s}$ (J2000 coordinates extracted from VLA FIRST Survey [3]).

IC 2497 was observed for two hours with the European VLBI Network (EVN) using the eVLBI technique at $\lambda 18 \mathrm{~cm}$, with a data rate of $512 \mathrm{Mbps}$ on the 30th September 2008, and for 10 hours on the 19th May 2009 at $\lambda 18 \mathrm{~cm}$ with a data rate of $1 \mathrm{Gbps}$. The first observation was taken as part of a multi-wavelength radio survey of IC 2497 and Hanny's Voorwerp [2]. In both observations the target was phase-referenced to J0945+3534, a calibrator 1.3 degrees away, figures $1 \mathrm{a}$ and $1 \mathrm{~b}$ show the UV coverage for both observations. The calibration solutions derived from J0945+3534 (including phase and amplitude corrections obtained by hybrid mapping the source) were applied to the IC 2497 data.

A compact radio source (hereafter $\mathrm{C} 1$ ) was detected from the phase calibrated data set of the first short e-VLBI observation, using natural weighting (Figure 2a). The observed position of this compact radio source (RA $094104.0872 \pm 0.0001$ and Dec. $344357.7776 \pm 0.0014$, J2000) is offset by approximately 230 milliarcseconds to the South-West of the VLA FIRST position. The output from the AIPS task IMFIT suggests an image size of $<60$ milliarcseconds and a flux density of $S_{1.65 \mathrm{GHz}, \mathrm{VLBI}}=1.09 \pm 0.14 \mathrm{mJy}$. From this we measure a minimum brightness temperature of $\sim 4 \times 10^{5} \mathrm{~K}$.

For the second much longer, e-VLBI observation, the target was phase referenced and IC 2497 was then phase self-calibrated with a long solution interval of 10 minutes averaging across the entire $128 \mathrm{MHz}$ band. A weighted image of robust $=2$ is shown in Figure 2b. The image confirms

\footnotetext{
1'Voorwerp' is the Dutch word for "object"
} 

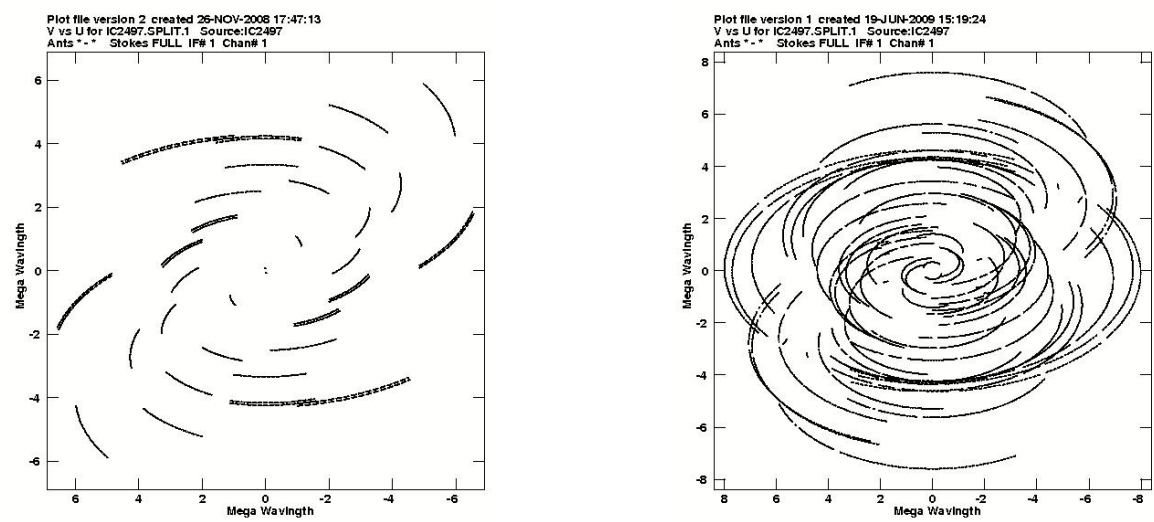

Figure 1: Left: IC 2497 was initially observed with the e-VLBI at $\lambda 18 \mathrm{~cm}$, for approximately 2 hours on the 30th September 2008, with 7 telescopes (Wb, Mc, On, Tr, Ef, Jb(MkII) and Da.) Right: The second $\lambda$ $18 \mathrm{~cm}$ eVLBI observation, took place on the 19th May 2009, and included 10 telescopes (Ef, Mc, On, Tr, $\mathrm{Wb}, \mathrm{Cm}, \mathrm{Jb}$ (Lovell), Da, De, \& Kn. ). Total observing time was 10 hours.

the existence of $\mathrm{C} 1$, but it also reveals the existence of another compact component to the NorthEast of C1. The second radio source (hereafter C2) has a flux density of $S_{1.65 \mathrm{GHz}, \mathrm{VLBI}}=0.594 \pm$ $0.004 \mathrm{mJy}$, and for $\mathrm{C} 1$ we measure a flux density of $S_{1.65 \mathrm{GHz}, \mathrm{VLBI}}=1.020 \pm 0.003 \mathrm{mJy}$. For C2, we determine a minimum brightness temperature of $1.4 \times 10^{5} \mathrm{~K}$.

\subsection{MERLIN observations}

On the 2nd of February 2009, IC 2497 was further observed at $\lambda 18 \mathrm{~cm}$ with the Multi-Element Radio Linked Interferometer Network (MERLIN). The MERLIN data was phase-calibrated to $0942+358 \mathrm{~B}$, and the phase solutions derived were transferred to the target. No self-calibration techniques were applied as the source was very weak and heavily resolved on the Cambridge-Defford baseline. The naturally weighted image is shown in Figure 3. The lower resolution MERLIN image shows a more complex radio morphology: both $\mathrm{C} 1$ and $\mathrm{C} 2$ are embedded in extended emission that flares out along the direction to the Voorwerp and also perpendicular to this direction.

\section{Discussion}

Our observations further support the hypothesis that IC 2497 contains an obscured AGN at its core [2]. In this scenario the Voorwerp is illuminated by the AGN after a jet clears a path from the AGN towards the Voorwerp. From our e-VLBI and MERLIN observations we detect 2 compact radio sources ( $\mathrm{C} 1$ and $\mathrm{C} 2$ ), at the center of IC 2497. From the e-VLBI observations we measure the minimum brightness temperature of both $\mathrm{C} 1$ and $\mathrm{C} 2$ to be $4 \times 10^{5} \mathrm{~K}$, and $1.4 \times 10^{5} \mathrm{~K}$, respectively. The brightness temperature associated with both components, suggest that they are related to AGN activity.

It is possible we may be observing an AGN core and a hotspot associated with the jet responsible for the Voorwerp. However, from our observations it is very difficult to determine which of the 

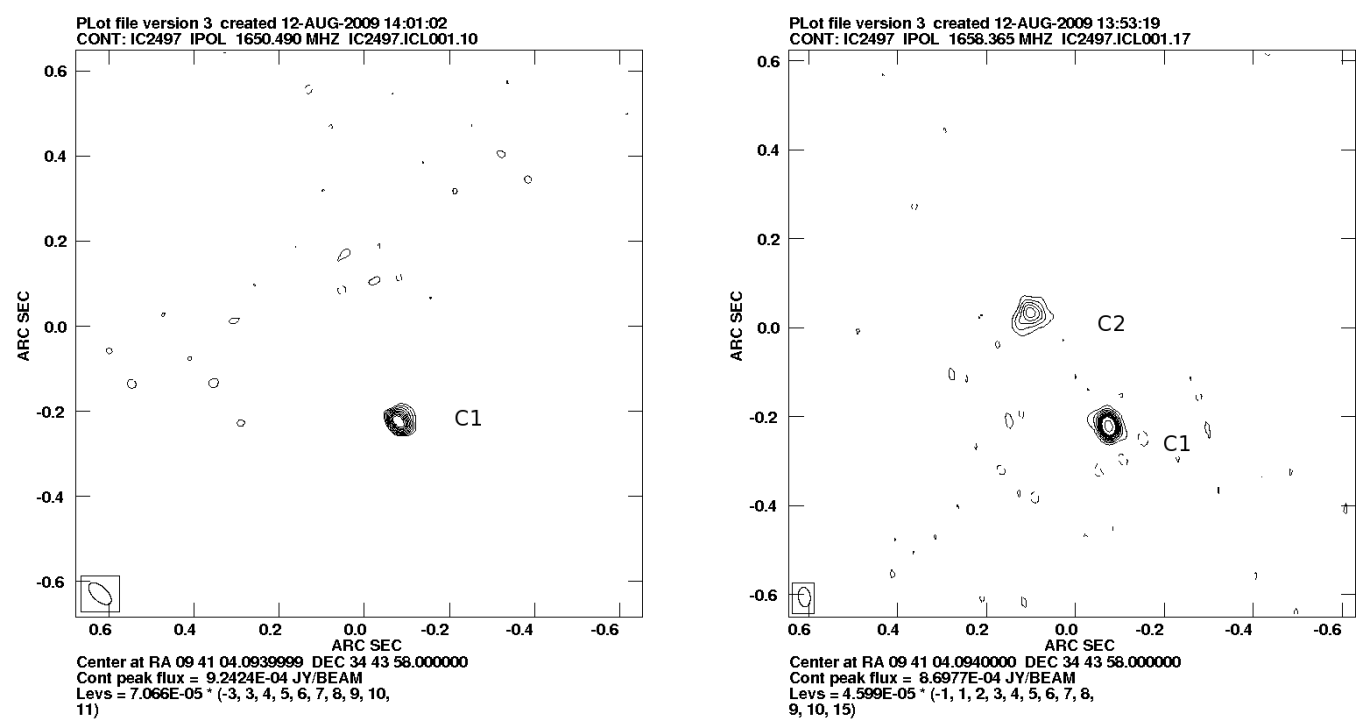

Figure 2: Left: $\lambda 18 \mathrm{~cm}$ e-VLBI radio map of IC 2497 on 2008 September 2008, with the component C1. The contours are at $-3,3,4,5,6,7,8,9,10 \times 0.071 \mathrm{mJybeam}^{-1}$, with a rms of $0.076 \mathrm{mJy}^{\mathrm{beam}}{ }^{-1}$. The beam size is $58 \times 35 \mathrm{mas}^{2}$ at P.A. $=49.6^{\circ}$. Right: $\lambda 18 \mathrm{~cm} \mathrm{e-VLBI}$ radio map of IC 2497 on 2009 May 19th, showing both components, $\mathrm{C} 1 \& \mathrm{C} 2$. The contours are at $-1,1,2,3,4,5,6,7,8,9,10,15 \times 0.046 \mathrm{mJybeam}^{-1}$, with an rms of $0.015 \mathrm{mJy}_{\text {beam }}{ }^{-1}$. The beam size is $45 \times 27 \mathrm{mas}^{2}$ at P.A. $=7.7^{\circ}$.

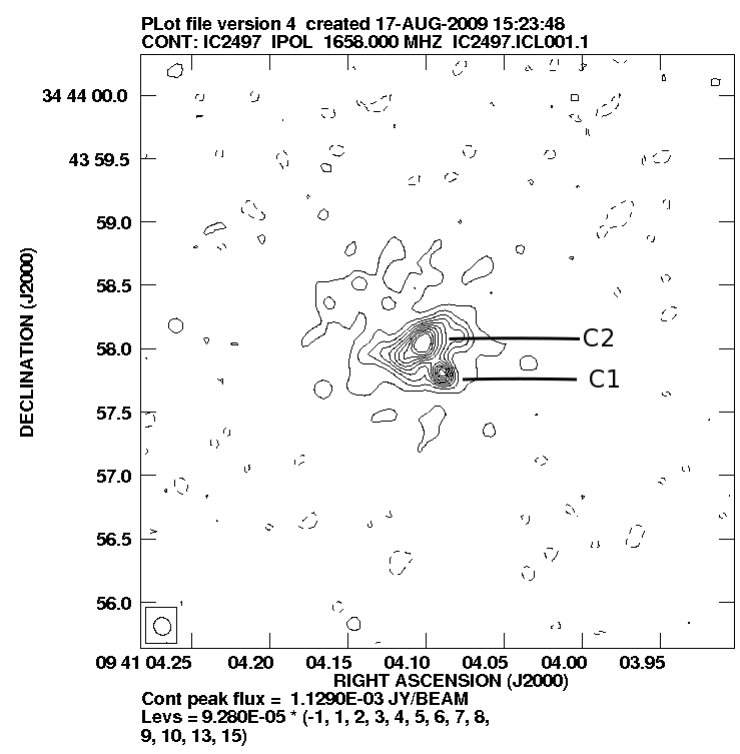

Figure 3: $\lambda 18 \mathrm{~cm}$ MERLIN radio map of IC 2497 on 2009 February 02 , showing both C1 \& C2, embedded within extended emission. The contours are at $-1,2,2,3,4,5,6,7,8,9,10,11,12,13,14,15 \times 0.092 \mathrm{mJybeam}^{-1}$, with an rms $0.037 \mathrm{mJy}_{\text {beam }}{ }^{-1}$. The beam size is $181 \times 162 \mathrm{mas}^{2}$, with a P.A. $=20.7^{\circ}$. 
components is the core. Higher frequency observations with MERLIN may be able to differentiate between the two via the spectral index of the components.

Another possible scenario is that the components $\mathrm{C} 1$ and $\mathrm{C} 2$ may be radio supernovae ( $\mathrm{RSNe}$ ). In comparison to the radio luminosities of the brightest RSNe from Arp 220 at $18 \mathrm{~cm}$ [5], we see that $\mathrm{C} 1$ is 6 times more luminous while $\mathrm{C} 2$ is only 3 times more luminous. From this we cannot exclude the possibility that $\mathrm{C} 2$ may be a RSNe.

The non-detection of $\mathrm{C} 2$ in the short EVN observation, may not necessarily indicate variability of the source at $18 \mathrm{~cm}$. Although the flux density of $\mathrm{C} 2$ is $\sim$ half of $\mathrm{C} 1$, most of the flux is in extended emission, which could not be recovered in the short EVN observation. The peak brightness of $\mathrm{C} 2$ is $0.236 \mathrm{mJy} / \mathrm{beam}$, which is at the 3 sigma rms noise level of the first EVN

observation. Note in the second EVN observation the rms is 0.0015 mJybeam $^{-1}$, which is $\sim 5$ times lower than the rms of the first EVN observation.

\section{Acknowledgements}

This research was supported by the EU Framework 6 Marie Curie Early Stage Training programme under contract number MEST-CT-2005-19669 "ESTRELA". e-VLBI developments in Europe are supported by the EC DG-INFSO funded Communication Network Developments project 'EXPReS', Contract No. 02662. The European VLBI Network is a joint facility of European, Chinese, South African and other radio astronomy institutes funded by their national research councils. MERLIN is a National Facility operated by the University of Manchester at Jodrell Bank Observatory on behalf of STFC.

\section{References}

[1] C. Lintott, K. Schawinski and W. Keel et al, Galaxy Zoo : 'Hanny's Voorwerp', a quasar light echo?., arXiv: 0906.5304L (2009)

[2] G. I. G. Jozsa and M. A. Garrett and T. A. Oosterloo and H. Rampadarath et al., Revealing Hanny's Voorwerp: radio observations of IC 2497, A\&A $\mathbf{5 0 0}$ (2009) 33.

[3] R. H. Becker, R. L. White and D. J. Helfand, The FIRST Survey: Faint Images of the Radio Sky at Twenty Centimeters., ApJ 450 (1995) 559

[4] K. B. Fisher, J. P. Huchra, M. A. Strauss, M. Davis, A. Yahil and D. Schlegel., The IRAS 1.2 Jy Survey: Redshift Data, ApJ Supplement Series 100 (1995) 69

[5] R. Parra, J. E. Conway, P. J. Diamond, H. Thrall, C. J. Lonsdale, C. J. Lonsdale, and H. E Smith., The Radio Spectra of the Compact Sources in Arp 220: A Mixed Population of Supernovae and Supernova Remnants ApJ $\mathbf{6 5 9}$ (2007) 314 